\title{
TRANSVERSE SHEAR MODULUS OF ELASTICITY FOR THIN-WALLED CORRUGATED CORES OF SANDWICH BEAMS. THEORETICAL STUDY
}

\author{
Ewa Magnucka-Blandzi \\ Poznan University of Technology, Institute of Mathematics, Poznan, Poland \\ e-mail: ewa.magnucka-blandzi@put.poznan.pl \\ KrZYSZTOF MAGNUCKI \\ Poznan University of Technology, Institute of Applied Mechanics, Poznań, Poland \\ e-mail: krzysztof.magnucki@put.poznan.pl
}

\begin{abstract}
The subject of the paper are four corrugated cores in form of circular arcs, a sin wave, trapezoids and an odd function. Transverse shear modules of these corrugated cores are analytically determined. A comparative analysis of these transverse shear modules is presented. Areas of cross sections of the corrugated cores are constant. The theoretical study shows considerable sensitivity of the shear modulus to shape of the corrugation.
\end{abstract}

Keywords: shear modulus, corrugated core, sandwich beams

\section{Introduction}

Theoretical fundamentals for sandwich structures were initiated in the mid of the 20th century. The shear modulus of a core of a sandwich structure considerably affects its bending and buckling. Libove and Hubka (1951) presented the primary elaboration related to analytical study of elastic constants for corrugated cores of sandwich plates. Carlsson et al. (2001) reviewed and adapted previous analytical approaches to the analysis of elastic stiffnesses of corrugated core sandwich panels into the framework of the first-order shear deformation laminated plate theory. Buannic et al. (2003) computed the effective properties of corrugated core sandwich panels using homogenisation theory. Motivated by the results of numerical simulations, Aboura et al. (2004) examined behaviour of the linear homogeneous cardboard analytically, and confirmed good agreeement of the results. A numerical approach to evaluate the stiffness parameters for corrugated board was described by Biancolini (2005). Cheng et al. (2006) proposed finite element analysis approach to evaluate equivalent elastic properties of complex sandwich structures. The elastic bending of unstiffened and stiffened corrugated plates was studied by Peng et al. (2007), where a mesh-free Galerkin method was applied in the analysis. A method for the modelling of a corrugated board panel was outlined by Isaksson et al. (2007) - corrugated core sandwiches were homogenized and the panels transformed to equivalent homogeneous layers with effective equal properties. Talbi et al. (2009) presented an analytical homogenization model for a corrugated cardboard and its numerical implementation with a shell element. An analytical model for the compressive and shear response of monolithic and hierarchical corrugated composite cores was developed by Kazemahvazi and Zenkert (2009). Kress and Winkler (2010) studied the problem of finding a substitute material model for describing the load response of globally flat corrugated sheets made of multidirectional laminates. They determined the maximal possible deformations of a corrugated sheet where the corrugation pattern consisted of two circular segments. Pan et al. (2008) investigated the transverse shear mechanical behaviour and failure mechanism of aluminum alloy honeycomb. He et al. (2012) presented a semi-analytical method for bending analysis of the sandwich panel with a core of triangular-shape, honeycomb-shape and X-shape. 
Aganovic et al. (1996) presented the equilibrium displacements corresponding to Koiter's shell model. The sequence of shells was considered as a slight periodical perturbation of the middle surface of the plate was shown to converge to the equilibrium displacement of the classical plate model. Corresponding corrector-type results were proved by the homogenization method. Michalak (2001) presented such a form of the mezo-shape function for a mezostructural model, which is suitable for quantitative analysis of dynamic behaviour of a wavy-plate. Governing equations of the averaged theory of wavy-plates were obtained for different forms of the mezo-shape functions for in-plane and out-of-plane displacements of the plate. The work does not address the averaged values of modules determined, for example, with the use the averaged theory or the asymptotic homogenization method, which are presented by Aganivic et al. and by Michalak.

The subject of the theoretical study presented in this paper are four corrugated cores in form of circular arcs, a sin wave, trapezoids and an odd function. The transverse shear modulus for each core is analytically determined.

\section{Analytical description of shear moduli for corrugated cores}

\subsection{Corrugation of the core in form of circular arcs}

The corrugated core between two faces undergoes shearing as shown in Fig. 1.

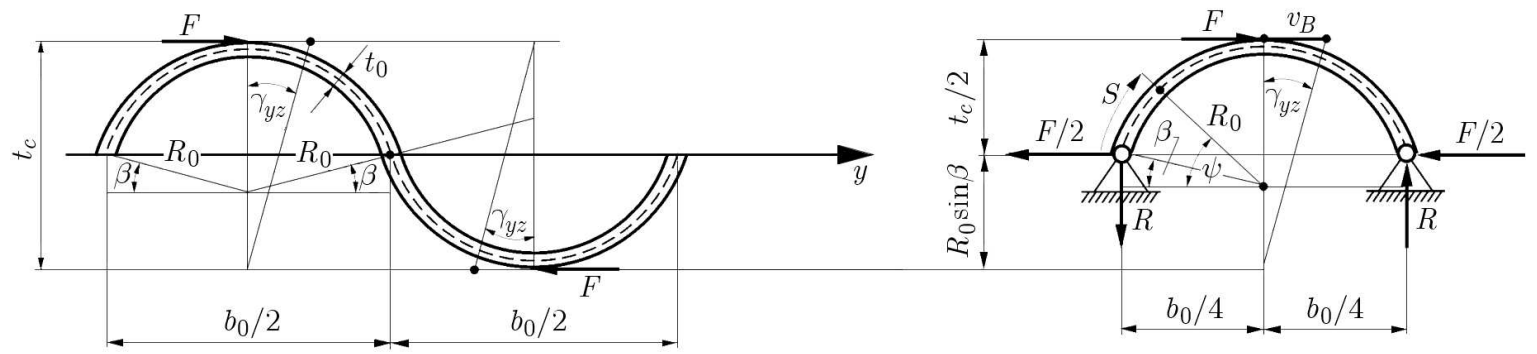

Fig. 1. Scheme of the corrugated core of the circular arcs shape

Geometrical relations for the circular arc (Fig. 1) are as follows:

— radius of the circular arc

$$
R_{0}=\frac{t_{c}}{16 C_{c a}}
$$

— complementary angle of the circular arc

$$
\beta=\arccos \left(4 x_{b 0} C_{c a}\right) \quad \text { for } \quad 2\left(1-x_{t 0}\right) \leqslant x_{b 0}
$$

where: $C_{c a}=\left(1-x_{t 0}\right) /\left[x_{b 0}^{2}+4\left(1-x_{t 0}\right)^{2}\right], x_{t 0}=t_{0} / t_{c}, x_{b 0}=b_{0} / t_{c}$ - dimensionless parameters, $b_{0}-$ corrugation pitch.

The basic system of forces for the half-pitch of the circular arc corrugation (Fig. 1) with the reaction

$$
R=\frac{1}{x_{b 0}} F
$$

enables one to formulate the bending moment

$$
M_{b}^{(c a)}(\varphi)=\frac{1}{2} F R_{0}\left[\sin \varphi-\sin \beta-\frac{2}{x_{b 0}}(\cos \beta-\cos \varphi)\right]
$$


The elastic strain energy

$$
U_{\varepsilon}^{(c a)}=\frac{12 R_{0}}{E a t_{0}^{3}} \int_{\beta}^{\pi / 2}\left[M_{b}^{(c a)}(\varphi)\right]^{2} d \varphi
$$

where $a$ is width of the corrugated core in the $\mathrm{x}$-axis direction.

The displacement $v_{B}$ (Fig. 1) is determined on the basis of Castigliano's second theorem

$$
v_{B}=\frac{d U_{\varepsilon}^{(c a)}}{d F}=\frac{6 F R_{0}^{3}}{E a t_{0}^{3}} S_{c a}
$$

where

$$
\begin{aligned}
& S_{c a}=S_{c a 1}+\frac{4}{x_{b 0}} S_{c a 2}+\frac{4}{x_{b 0}^{2}} S_{c a 3} \\
& S_{c a 1}=\left(\frac{\pi}{2}-\beta\right)\left(\frac{1}{2}+\sin ^{2} \beta\right)-\frac{3}{4} \sin (2 \beta) \\
& S_{c a 2}=1-\sin \beta+\frac{1}{2}\left(\frac{\pi}{2}-\beta\right) \sin (2 \beta)-\frac{3}{2} \cos ^{2} \beta \\
& S_{c a 3}=\left(\frac{\pi}{2}-\beta\right)\left(\frac{1}{2}+\cos ^{2} \beta\right)-2\left(1-\frac{3}{4} \sin \beta\right) \cos \beta
\end{aligned}
$$

The shear strain in the $y z$-plane is as follows

$$
\gamma_{y z}^{(c a)}=\frac{v_{B}}{t_{c}}=\frac{6 F R_{0}^{3}}{E a t_{c} t_{0}^{3}} S_{c a}
$$

From Hooke's law

$$
\tau_{y z}^{(c a)}=\frac{F}{a b_{0}}=G_{y z}^{(c a)} \gamma_{y z}^{(c a)}
$$

the shear modulus of elasticity for the circular arc corrugation is

$$
G_{y z}^{(c a)}=\widetilde{G}_{y z}^{(c a)} E
$$

where the dimensionless shear modulus is

$$
\widetilde{G}_{y z}^{(c a)}=\frac{2048}{3} \frac{x_{t 0}^{3} C_{c a}^{3}}{x_{b 0} S_{c a}}
$$

The cross section area of the circular arc corrugation for one pitch (Fig. 1) amounts to

$$
A_{0}^{(c a)}=2(\pi-2 \beta) R_{0} t_{0}=\widetilde{A}_{0}^{(c a)} t_{c}^{2}
$$

where the dimensionless area

$$
\widetilde{A}_{0}^{(c a)}=\frac{1}{8}(\pi-2 \beta) \frac{x_{t 0}}{C_{c a}}
$$




\subsection{Corrugation of the core in form of a $\sin$ wave}

The function of the corrugation is

$$
f(y)=\frac{1}{2} t_{c}\left(1-x_{0}\right) \sin (2 \pi \eta)
$$

where $\eta=y / b_{0}$ denotes the dimensionless coordinate.

The force system for the half-pitch of the sin wave corrugation (Fig. 2) is similar to that of the circular arc corrugation with reaction (2.3). The bending moment is

$$
M_{b}^{(\sin )}(\eta)=\frac{1}{4} F t_{c}\left[\left(1-x_{t 0}\right) \sin (2 \pi \eta)-4 \eta\right]
$$

Then, the elastic strain energy

$$
U_{\varepsilon}^{(c a)}=\frac{12 b_{0}}{E a t_{0}^{3}} \int_{0}^{1 / 4}\left[M_{b}^{(\sin )}(\eta)\right]^{2} \sqrt{1+c_{0}^{2} \cos ^{2}(2 \pi \eta)} d \eta
$$

where $c_{0}=\pi\left(1-x_{t 0}\right) / x_{b 0}$ is the dimensionless parameter.
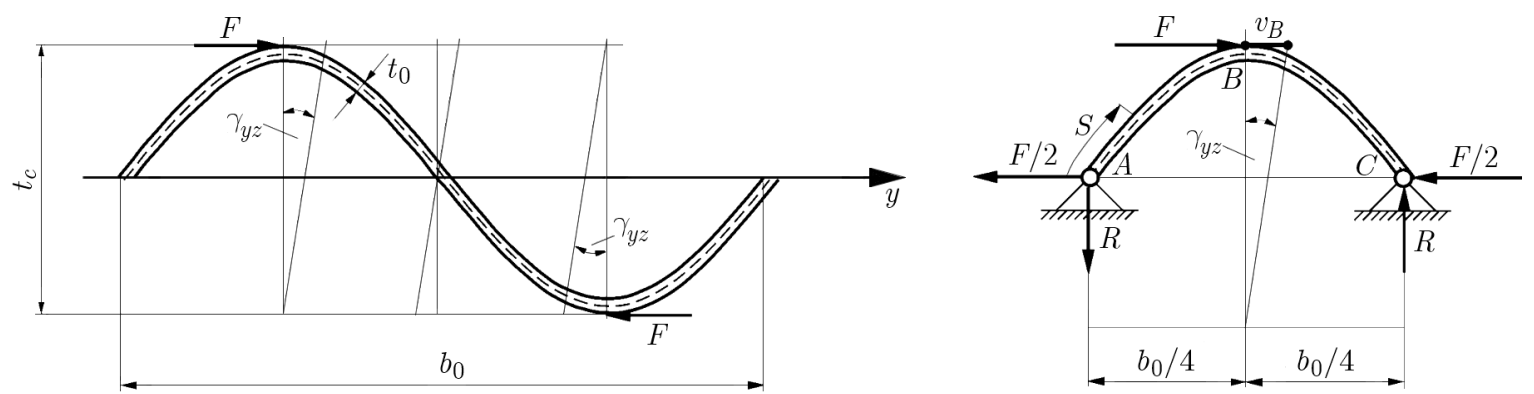

Fig. 2. Scheme of the wave-shaped corrugated core

The displacement $v_{B}$ (Fig. 2) on the basis of Castigliano's second theorem is as follows

$$
v_{B}=\frac{d U_{\varepsilon}^{(\sin )}}{d F}=\frac{3 F b_{0} t_{c}^{2}}{2 E a t_{0}^{3}} S_{1}^{(\sin )}
$$

where

$$
\begin{aligned}
S_{1}^{(\sin )}= & \left(1-x_{t 0}\right)^{2} S_{11}^{(\sin )}+8\left(1-x_{t 0}\right) S_{12}^{(\sin )}+16 S_{13}^{(\sin )} \\
S_{11}^{(\sin )}= & \int_{0}^{1 / 4} \sin ^{2}(2 \pi \eta) \sqrt{1+c_{0}^{2} \cos ^{2}(2 \pi \eta)} d \eta \\
S_{12}^{(\sin )}= & \int_{0}^{1 / 4} \eta \sin (2 \pi \eta) \sqrt{1+c_{0}^{2} \cos ^{2}(2 \pi \eta)} d \eta \\
S_{13}^{(\sin )}= & \int_{0}^{1 / 4} \eta^{2} \sqrt{1+c_{0}^{2} \cos ^{2}(2 \pi \eta)} d \eta
\end{aligned}
$$

The shear strain in the $y z$-plane is as follows

$$
\gamma_{y z}^{(\sin )}=\frac{v_{B}}{t_{c}}=\frac{3 F b_{0} t_{0}}{2 E a t_{0}^{3}} S_{1}^{(\sin )}
$$


Thus, by analogy to expressions (2.8) and (2.9), the dimensionless shear modulus is

$$
\widetilde{G}_{y z}^{(\sin )}=\frac{2 x_{t 0}^{2}}{3 x_{b 0}^{2} S_{1}^{(\sin )}}
$$

The cross section area of the circular arc corrugation for one pitch (Fig. 2) is

$$
A_{0}^{(\sin )}=4 b_{0} t_{0} S_{0}^{(\sin )}=\widetilde{A}_{0}^{(\sin )} t_{c}^{2}
$$

where

$$
S_{0}^{(\sin )}=\int_{0}^{1 / 4} \sqrt{1+c_{0}^{2} \cos ^{2}(2 \pi \eta)} d \eta
$$

and the dimensionless area

$$
\widetilde{A}_{0}^{(\sin )}=4 x_{t 0} x_{b 0} S_{0}^{(\sin )}
$$

\subsection{Corrugation of the core in form of trapezoids}

Geometrical relations for the trapezoid (Fig. 3) are as follows

$$
\sin \alpha_{0}=\frac{2\left(1-x_{t 0}\right)}{\sqrt{C_{t}}} \quad \cos \alpha_{0}=\frac{x_{b 0}-2 k_{b} x_{t 0}}{\sqrt{C_{t}}}
$$

where $k_{b}=b_{1} / t_{0}, C_{t}=\left(x_{b 0}-2 k_{b} x_{t 0}\right)^{2}+4\left(1-x_{t 0}\right)^{2}$ are dimensionless parameters.

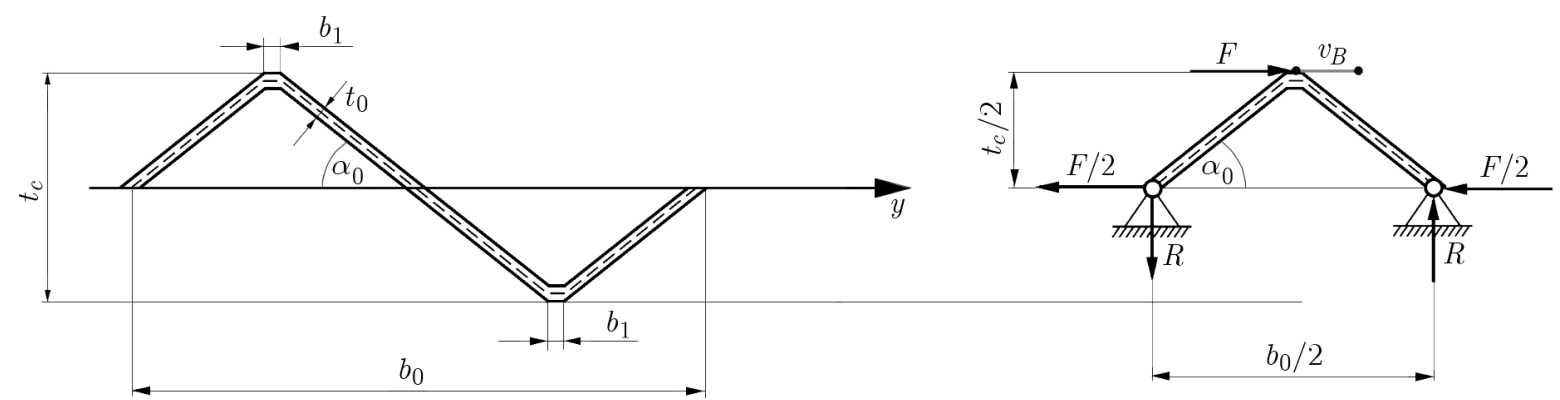

Fig. 3. Scheme of the corrugated core of the trapezoid shape

The force system for the half-pitch of the sin wave corrugation (Fig. 3) is similar to that of the circular arc corrugation with the reaction (2.3). The normal force and the bending moment in the trapezoidal corrugated core are

$$
\begin{aligned}
& N^{(\text {trap })}(s)=\frac{1}{2} F\left(\cos \alpha_{0}+\frac{2}{x_{b 0}} \sin \alpha_{0}\right) \\
& M_{b}^{(t r a p)}(s)=\frac{1}{2} F\left(\sin \alpha_{0}-\frac{2}{x_{b 0}} \cos \alpha_{0}\right) s
\end{aligned}
$$

The elastic strain energy with consideration of the tension and bending energy is as follows

$$
U_{\varepsilon}^{(\text {trap })}=\frac{1}{E a t_{0}} \int_{0}^{s_{t}}\left[N^{(\text {trap })}(s)\right]^{2} d s+\frac{12}{E a t_{0}^{3}} \int_{0}^{s_{t}}\left[M_{b}^{(\text {trap })}(s)\right]^{2} d s
$$

where $s_{t}=t_{c} \sqrt{C_{t}} / 4$ is the length of the trapezoid arm. 
The shear strain in the $y z$-plane, by analogy to expressions (2.6) or (2.16), is written

$$
\gamma_{y z}^{(\text {trap })}=\frac{v_{B}}{t_{c}}=\frac{F s_{t} t_{c}}{2 E a t_{0} b_{0}^{2}} S_{1}^{(\text {trap })}
$$

where

$$
S_{1}^{(\text {trap })}=\frac{1}{C_{t}} x_{b 0}\left[x_{b 0}\left(x_{b 0}-2 k_{b} x_{t 0}\right)+4\left(1-x_{t 0}\right)\right]+\left(x_{b 0}-2 k_{b}\right)^{2}
$$

Thus, the dimensionless shear modulus is

$$
\widetilde{G}_{y z}^{(t r a p)}=\frac{8 x_{t 0} x_{b 0}}{S_{1}^{(\sin )} \sqrt{C_{t}}}
$$

The cross section area of the trapezoid corrugation for one pitch (Fig. 3) is

$$
A_{0}^{(\text {trap })}=t_{c} t_{0}\left(2 k_{b} x_{t 0}+\sqrt{C_{t}}\right)=\widetilde{A}_{0}^{(t r a p)} t_{c}^{2}
$$

where the dimensionless area is

$$
\widetilde{A}_{0}^{(t r a p)}=x_{t 0}\left(2 k_{b} x_{t 0}+\sqrt{C_{t}}\right)
$$
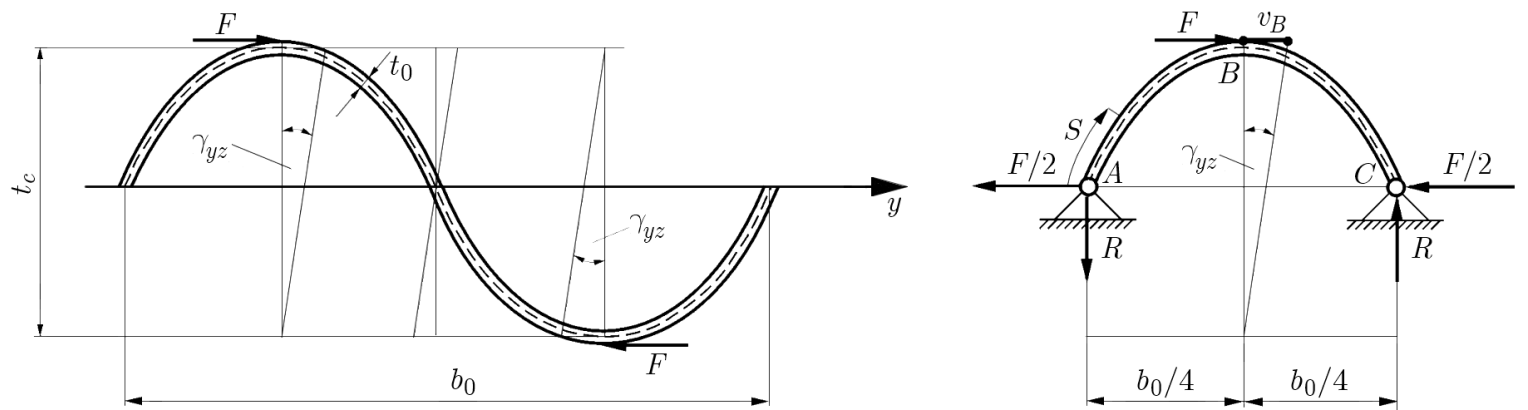

Fig. 4. Scheme of the corrugated core in form of an odd function shape

The function of the corrugation is

$$
f(y)=\frac{1}{2} t_{c}\left(1-x_{t 0}\right) \phi(\eta)
$$

where the odd function is in the following form

$$
\phi(\eta)=\eta\left[6-32 \eta^{2}+\frac{k_{f}}{256}\left(1-32 \eta^{2}+256 \eta^{4}\right)\right]
$$

and $k_{f}$ is dimensionless parameter.

Thus, by analogy to the sin wave corrugation the dimensionless shear modulus is

$$
\widetilde{G}_{y z}^{(o d d-f)}=\frac{2 x_{t 0}^{2}}{3 x_{b 0}^{2} S_{1}^{(o d d-f)}}
$$

where

$$
\begin{aligned}
& S_{1}^{(\text {odd }-f)}=\int_{0}^{1 / 4}\left[\left(1-x_{t 0}\right) \phi(\eta)-4 \eta\right]^{2} \sqrt{1+c_{f}^{2} \phi_{1}^{2}(\eta)} d \eta \\
& c_{f}=\frac{1-x_{t 0}}{2 x_{b 0}} \quad \phi_{1}(\eta)=6-96 \eta^{2}+\frac{k_{f}}{256}\left(1-96 \eta^{2}+1280 \eta^{4}\right)
\end{aligned}
$$


and the dimensionless area

$$
\widetilde{A}_{0}^{(o d d-f)}=4 x_{t 0} x_{b 0} S_{0}^{(o d d-f)}
$$

where

$$
S_{0}^{(o d d-f)}=\int_{0}^{1 / 4} \sqrt{1+c_{f}^{2} \phi_{1}^{2}(\eta)} d \eta
$$

Expressions (2.10), (2.18), (2.25) and (2.30) for the dimensionless shear moduli and (2.12), (2.20), (2.27) and (2.31) for dimensionless areas serve as a basis of comparative analysis of the four shapes of the corrugated cores.

\section{Comparative analysis of shear moduli of the corrugated cores}

Values of deflections and critical loads of sandwich structures are related to the values of the core shear moduli. Maximization of the value of the shear modulus results in the maximum value of rigidity of the sandwich structure. In consequence, the quality measure of the corrugated core is the value of the dimensionless shear modulus for a constant value of the dimensionless area of a single pitch. The comparative analysis is carried out for the following example data: thickness of the core $t_{c}=12.2 \mathrm{~mm}$, corrugation pitch $b_{0}=28 \mathrm{~mm}$ and dimensionless area of the single corrugation pitch $\widetilde{A}_{0}^{(c)}=0.2$. The geometric size and dimensionless transverse shear moduli of the studied cores calculated based on the above data are as follows:

- the circular arc shape of the corrugation (Fig. 1)

(Thickness of the corrugated sheet $t_{0}=0.760 \mathrm{~mm}$, radius of the circular arcs (2.1) $R_{0}=7.14 \mathrm{~mm}$, complementary angle $(2.2) \beta=0.2006 \mathrm{rad}$, and dimensionless shear modulus (2.10) $\widetilde{G}_{y z}^{(c a)}=0.00170$.)

- the sin wave shape of the corrugation (Fig. 2)

(The tickness of the corrugated sheet $t_{0}=0.799 \mathrm{~mm}$, and the dimensionless shear modulus (2.18) $\widetilde{G}_{y z}^{(\sin )}=0.00851$.)

- the trapezoid shape of the corrugation (Fig. 3)

(The maximum value of the dimensionless shear modulus $\widetilde{G}_{y z, \max }^{(t r a p)}=0.1755$ occurs for the thickness of the corrugated sheet $t_{0}=0.815 \mathrm{~mm}$ and the length of trapezoid parallel sides $b_{1}=0.9271 \mathrm{~mm}$.)

- the odd function shape of the corrugation (Fig. 4).

The maximum value of the dimensionless shear modulus $\widetilde{G}_{y z, \max }^{(\text {odd }}=0.2707$ occurs for the thickness of the corrugated sheet $t_{0}=0.816 \mathrm{~mm}$ and the dimensionless parameter $k_{f}=-507.9$. It can be noticed that for core corrugations in sin wave and odd function shapes, the values of the shear moduli are equal to $\widetilde{G}_{y z}^{(\sin )}=\widetilde{G}_{y z}^{(o d d-f)}=0.00851$ for the sheet thickness $t_{0}=0.799 \mathrm{~mm}$. Moreover, for the trapezoidal corrugation and sheet thickness $t_{0}=0.780 \mathrm{~mm}$, the value of the shear modulus $\widetilde{G}_{y z}^{(t r a p)}=0.00849$ approximates the above values of $\widetilde{G}_{y z}^{(\sin )}=\widetilde{G}_{y z}^{(\text {odd }-f)}=0.00851$. In this case, the graph of the sinusoidal shape of core corrugation coincides with the graph of the odd function (Fig. 5).

The shear moduli of the corrugated cores with circular arcs or sin wave shapes for any data are constant $\left(\widetilde{G}_{y z}^{(c a)}=0.00170, \widetilde{G}_{y z}^{(\sin )}=0.00851\right.$ are valid for the examplary data). The shapes of these corrugations are uniquely defined for the assumed data. However, the trapezoid or the 


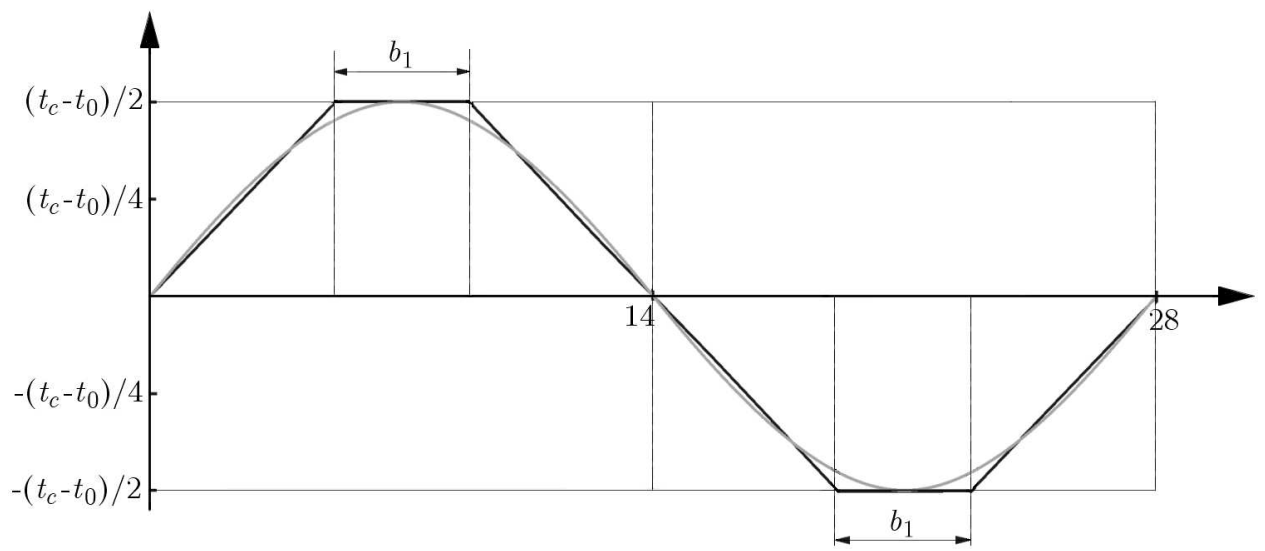

Fig. 5. Comparison of three corrugation shapes for the trapezoid $\left(b_{1}=3.710 \mathrm{~mm}\right)$, odd function and sin wave

odd function shapes are not uniquely defined for the assumed data as their shapes may be controlled by varying the length of the trapezoid parallel sides $b_{1}$ (Fig. 3) or the dimensionless parameter $k_{f}$ of the function (2.29). The results of numerical calculations for these shapes of corrugations (Table 1 and Table 2) are shown in Fig. 6.

Table 1. Values of $t_{0}, b_{1}$ and dimensionless shear modulus (2.25)

\begin{tabular}{|l|c|c|c|c|c|c|c|c|c|}
\hline$t_{0}[\mathrm{~mm}]$ & 0.780 & 0.790 & 0.800 & 0.805 & 0.810 & 0.8125 & 0.8150 & 0.8175 & 0.8251 \\
\hline$b_{1}[\mathrm{~mm}]$ & 3.710 & 2.973 & 2.192 & 1.783 & 1.362 & 1.1465 & 0.9271 & 0.7041 & 0 \\
\hline$\widetilde{G}_{y z}^{\text {trap })}$ & 0.00849 & 0.0152 & 0.0351 & 0.0622 & 0.1199 & 0.1574 & 0.1755 & 0.1552 & 0.0550 \\
\hline
\end{tabular}

Table 2. Values of $t_{0}, k_{f}$ and dimensionless shear modulus (2.30)

\begin{tabular}{|c|c|c|c|c|c|c|c|c|c|}
\hline$t_{0}[\mathrm{~mm}]$ & 0.799 & 0.810 & 0.812 & 0.813 & 0.814 & 0.815 & 0.8155 & 0.816 & 0.8164 \\
\hline$k_{f}[\mathrm{~mm}]$ & 71.36 & -199.3 & -270.0 & -311.4 & -359.4 & -418.7 & -456.7 & -507.9 & -607.6 \\
\hline$\widetilde{G}_{y z}^{\text {(odd }}$ (of) & 0.00851 & 0.0264 & 0.0397 & 0.0523 & 0.0748 & 0.1245 & 0.1769 & 0.2707 & 0.2526 \\
\hline
\end{tabular}

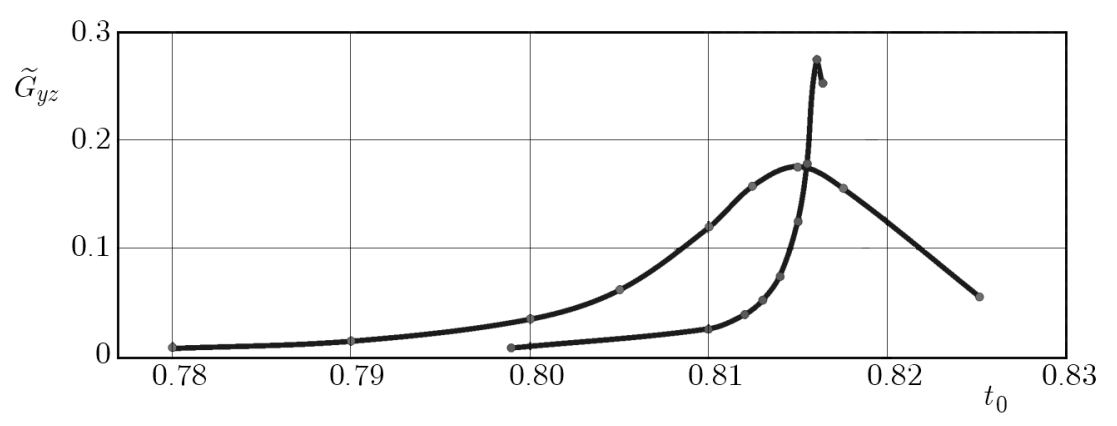

Fig. 6. Shear moduli for the trapezoid and odd function core shapes

These graphs reveal the shear moduli sensitivity to variation of the corrugated sheet thickness $t_{0}$ for the above two shapes of corrugations. A minor change in the of corrugated sheet thickness $t_{0}$ results in a significant change in the shear moduli values $\widetilde{G}_{y z}^{(t r a p)}$ and $\widetilde{G}_{y z}^{(o d d-f)}$. This sensitivity arises in the neighbourhood of the extremum. Thus, manufacturing of sandwich beams with corrugated cores of trapezoid or odd function shapes may be impossible, taking into account the maximum values of the shear moduli. The profile and dimensional tolerance is of high importance in this case. The trapezoid and odd function core shapes for the extremum (the 
trapezoid: $t_{0}=0.815 \mathrm{~mm}, b_{1}=0.9271 \mathrm{~mm}, \widetilde{G}_{y z, \max }^{(t r a p)}=0.1755$, the odd function: $t_{0}=0.816 \mathrm{~mm}$, $\left.k_{f}=-507.9, \widetilde{G}_{y z, \max }^{(o d d-f)}=0.2707\right)$ are shown in Fig. 7.

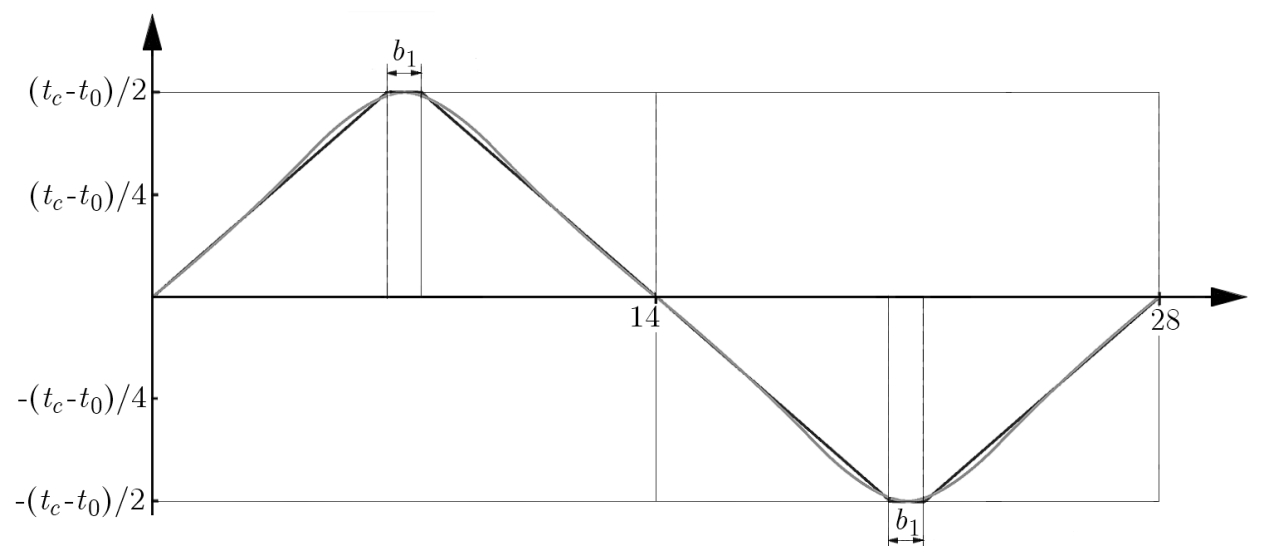

Fig. 7. Comparison of the two corrugation shapes for the trapezoid $\left(b_{1}=0.9271 \mathrm{~mm}\right)$ and the odd function at extremum



Fig. 8. Comparison of the three corrugation shapes for the trapezoid $\left(b_{1}=0.9271 \mathrm{~mm}\right)$ odd function and sine wave form

It can be noticed that in the extreme case, the shapes of core corrugations in of the trapezoid and odd function type are similar.

\section{Conclusions}

The theoretical studies of four corrugated cores allows one to draw the following conclusions:

- the core in form of circular arc is the most susceptible to shearing when the value of dimensionless transverse shear modulus of elasticity is the lowest: $\widetilde{G}_{y z}^{(c a)}=0.00170$,

- the core in form of the sin wave is more resistant to shearing than the circular arc core when the transverse shear modulus of elasticity is higher: $\widetilde{G}_{y z}^{(\sin )}=0.00851$,

- the trapezoidal core is much more resistant to shearing than the two above, the maximum value of the transverse shear modulus is $\widetilde{G}_{y z, \max }^{(t r a p)}=0.1755$, nevertheless, the shear modulus is sensitive to variation of the corrugated sheet thickness $t_{0}$,

- the core having shape of an odd function is distinguished by the greatest resistance to shearing, the maximum value of the transverse shear modulus is $\widetilde{G}_{y z, \max }^{(o d d-f)}=0.2707$, however, it is very sensitive to the change of the corrugated sheet thickness $t_{0}$. 
The theoretical studies show significant differences between the four shapes, including the three basic ones: circular arcs, sin wave and trapezoid.

Acknowledgements

The research was conducted within the framework of Statutory Activities No. 04/43/DSPB/0079 and $02 / 21 / \mathrm{DSPB} / 3452$.

\section{References}

1. Aboura Z., Talbi N., Allaoui S., Benzeggagh M.L., 2004, Elastic behavior of corrugated cardboard: experiments and modeling, Composite Structures, 63, 53-62

2. Aganovic I., Marusic-Paloka E., Tutek Z., 1996, Slightly wrinkled plate, Asymptotic Analysis, 13, 1-29

3. Biancolini M.E., 2005, Evaluation of equivalent stiffness properties of corrugated board, Composite Structures, 69, 322-328

4. Buannic N., Cartraud P., Quesnel T., 2003, Homogenization of corrugated core sandwich panels, Composite Structures, 59, 299-312

5. Carlsson L.A., Nordstrand T., Westerlind B., 2001, On the elastic stiffnesses of corrugated core sandwich, Journal of Sandwich Structures and Materials, 3, 253-267

6. Cheng Q.H., Lee H.P., Lu C., 2006, A numerical analysis approach for evaluating elastic constants of sandwich structures with various core, Composite Structures, 74, 226-236

7. He L., Cheng Y.-S., Liu J., 2012, Precise bending stress analysis of corrugated-core, honeycombcore and X-core sandwich panel, Composite Structures, 94, 1656-1668

8. Isaksson P., Krusper A., Gradin P.A., 2007, Shear correction factor for corrugated core structures, Composite Structures, 80, 123-130

9. Kazemahvazi S., Zenkert D., 2009, Corrugated all-composite sandwich structures. Part 1: Modeling, Composites Science and Technology, 69, 913-919

10. Kress G., Winkler M., 2010, Corrugate laminate homogenization model, Composite Structures, 92, 795-810

11. Libove C., Hubka R.E., 1951, Elastic constants for corrugated-core, Sandwich plates, Technical Note 2289, NACA, Washington

12. Michalak B., 2001, Analysis of dynamic behaviour of wavy-type plates with a mezzo-periodic structure, Journal of Theoretical and Applied Mechanics, 39, 947-958

13. PAn S.-D., Wu L.-Z., Sun Y.-G., 2008, Transverse shear modulus and strength of honeycomb cores, Composite Structures, 84, 369-374

14. Peng L.X., Liew K.M., Kitipornchai S., 2007, Analysis of stiffened corrugated plates based on the FSDT via the mesh-free method, International Journal of Mechanical Sciences, 49, 364-378

15. Talbi N., Batti A., Ayad R., Guo Y.Q., 2009, An analytical homogenization model for finite element modeling of corrugated cardboard, Composite Structures, 88, 280-289

16. Winkler M., Kress G., 2010, Deformation limits for corrugated cross-ply laminates, Composite Structures, 92, 1458-1468

Manuscript received January 6, 2014; accepted for print May 12, 2014 